\title{
Expression of HER2 and C-KIT in Nasopharyngeal Carcinoma: Implications for a New Therapeutic Approach
}

\author{
G. Bar-Sela, A. Kuten, S. Ben-Eliezer, E. Gov-Ari, O. Ben-Izhak \\ Departments of Oncology (GB-S, AK), Pathology (SB-E, OB-I), and Otolaryngology (EG-A), Rambam \\ Medical Center and Faculty of Medicine, Technion-Israel Institute of Technology, Haifa, Israel
}

\begin{abstract}
We sought to determine the expression and prognostic significance of HER2 and c-KIT proteins in nasopharyngeal carcinoma (NPC). In this retrospective study, immunohistochemical stains for HER2 and c-KIT were performed on formalin-fixed paraffinembedded sections from 49 patients with NPC who were treated at our hospital from 1971 to 2000. The clinical and immunohistochemical data were correlated, including gender, ethnic origin, age, histological type, EBV status (EBER in situ hybridization), stage, and overall survival. HER2 expression was not found in the tested samples. C-KIT overexpression was found in 33\% (16/49) of the patients. Nine of the 16 samples $(56 \%)$ were strongly positive for $\mathrm{c}$-KIT protein (staining of $>50 \%$ of the tumor cells). C-KIT expression was associated with younger age. C-KIT was not found in patients with squamous carcinoma or in those with negative EBV status, although these two groups consisted of only five patients each. Although c-KIT-positive cases tended to be associated with slightly better survival, this was not statistically significant. C-KIT protein was expressed in one third of the NPC patients in this study, only in EBV-positive, undifferentiated, or nonkeratinizing carcinoma patients. Further study is needed to check whether c-KIT expression is correlated with c-KIT DNA mutations and to test the possibility of treatment with imatinib mesylate (Gleevec). HER2 protein was negative in the same tested specimens.
\end{abstract}

KEY WORDS: C-KIT, HER2, Nasopharyngeal carcinoma.

Mod Pathol 2003;16(10):1035-1040

Copyright () 2003 by The United States and Canadian Academy of Pathology, Inc.

VOL. 16, NO. 10, P. 1035, 2003 Printed in the U.S.A.

Date of acceptance: June 20, 2003.

Address reprint requests to: G. Bar-Sela, Department of Oncology, Rambam Medical Center, POB 9602, Haifa 31096 Israel; fax: 972-4-854-2929; e-mail: bar_sela@rambam.health.gov.il.

DOI: 10.1097/01.MP.0000089778.48167.91
Nasopharyngeal carcinoma (NPC) has distinct epidemiological, pathological, and clinical characteristics among head and neck carcinomas. It is highly prevalent in southern China (1), and Israel is one of the world's intermediate risk areas for NPC, with most patients having Type III histology (undifferentiated carcinoma) and advanced stage on diagnosis (2). In contrast with other head and neck carcinomas, distant metastases develop in approximately $50 \%$ of all cases of NPC $(3,4)$. Because the tumor is radiosensitive, radiotherapy is the primary treatment modality. Although radiation therapy is highly effective in controlling locoregional disease, the majority of treatment failures are a result of distant metastases. The new treatment approach in locoregional Stage III and IV patients of radiotherapy with concurrent cisplatin followed by adjuvant chemotherapy has shown better progression-free survival and overall survival (5). This treatment is now considered as the standard treatment for locally advanced NPC. However, even within the same staging category, there will be variability in patient outcome, indicating the influence of other factors that affect tumor response to therapy.

HER2 (c-erbB-2) is a proto-oncogene that encodes a human epidermal cell growth factor receptor-like protein. In vitro and animal studies have suggested that HER2 gene amplification and protein overexpression might play a pivotal role in tumorigenesis (6). There is increased evidence that an HER2-positive status is of independent prognostic or predictive relevance in a variety of human cancers, including breast, ovarian, gastric, and non-small cell lung cancer (7-10). In NPC, there are conflicting data about the status of HER2 in the DNA level, the protein level, and the prognostic value of the findings (11-14).

C-KIT (CD117) is a transmembrane growth factor receptor for stem cell factor. It belongs to the family of Type III receptor tyrosine kinases and is structurally and functionally closely related to the platelet-derived growth factor receptor $(15,16)$. The 
C-KIT receptor is expressed in a limited variety of cell lineages, including mast cells, germ cells, interstitial cells of Cajal, and melanocytes $(17,18)$. Although weak immunohistochemical staining has been reported in a variety of tumors (17), strong C-KIT expression is largely limited to gastrointestinal stromal tumors (GIST) (18), mast cell disease (17), seminoma (19), few melanomas, and few serous ovarian carcinomas (17). To the best of our knowledge, c-KIT has not been studied in a large series of NPC patients.

The new cancer therapy directed at specific signaling pathways of cancer cells showed remarkable efficacy in HER2-positive advanced breast cancer patients with trastuzumab (Herceptin) treatment (20) or with imatinib mesylate (Gleevec) in c-KITpositive GIST (21). The possibility of treating other malignant tumors with those agents prompted this study of the expression of HER2 and c-KIT in NPC. Given these observations, we evaluated a cohort of NPC patients who were primarily treated with radiation or chemoradiation therapy from a single Israeli institution and correlated clinicopathological data with immunohistochemical staining for HER2 and c-KIT.

\section{MATERIALS AND METHODS}

\section{Patients}

A total of 244 patients with NPC treated at Rambam Medical Center in Haifa, Israel, from 1971 to 2000 were identified from the tumor registry records of the Department of Oncology. Inclusion criteria consisted of the availability of histologically confirmed, adequately sized tissue specimens of NPC that were available from the department of pathology in the same medical center. Follow-up records for a minimum period of 2 years after completion of treatment or until the diagnosis of recurrence were also required. Forty-nine cases were adequately available for immunohistochemical stains and were included in the study, and latest status was known for 47 patients (follow-up on 2 patients was not found). Treatment consisted of irradiation only or combined irradiation and chemotherapy. The patient's hospital charts and the national tumor registry records were reviewed to determine clinical stage, distant and nodal metastatic status, and survival. Clinical stage was determined according to the 1998 American Joint Committee of Cancer criteria, but complete clinical stage was found for only 37 patients. Data regarding recurrence were missing in some charts and, therefore, were not included in the statistical analysis. Survival statistical analysis was performed for the following: alive without disease (all with follow-up of $>5$ years), dead of disease, and dead without disease.

\section{Immunohistochemical Staining}

The tissue sections were deparaffinized in xylene twice for 10 minutes each and then rehydrated in graded alcohols and rinsed in tap water. Before immunostaining for HER2, the slides were microwaved at $92^{\circ} \mathrm{C}$ for 15 minutes in buffer EDTA ( $\mathrm{pH}=$ 8; Zymed Laboratory, San Francisco, CA), followed by cooling for 20 minutes at room temperature. All samples were stained in the Ventana immunostainer (NexES) according to manufacturer's instructions. For HER2, a polyclonal anti-HER2 antibody (DAKO, Glostrup, Denmark) at a dilution of 1:1100 was used. Heat-induced antigen retrieval was not performed before c-KIT immunostaining. For c-KIT, a polyclonal antibody (DAKO) at a dilution of 1:150 was used. Finally, the slides were counterstained with hematoxylin and mounted. For positive controls, a HER2-positive breast carcinoma and a c-KIT-positive GIST were used. Negative controls for all immunostainings were derived by substituting the primary antibody with normal rabbit serum.

All slides were evaluated without knowledge of the patient's clinical status. The positive tumor cells were graded according to the number of positive cells. Only moderate-to-strong complete membrane staining of $>10 \%$ of the cells was considered as positive HER2 staining. Only unequivocal cytoplasmic staining of $>10 \%$ of the cells was considered as positive c-KIT staining, which was further subdivided to staining of $10-50 \%$ of the cells (positive) and staining of $>50 \%$ of the cells (strongly positive).

Epstein-Barr virus (EBV) status was determined by EBER in situ hybridization, which was performed according to the manufacturer's instructions (Novocastra Laboratories, Newcastle, UK), including positive controls given by the manufacturer and negative controls in every sample stained without the specific probe.

\section{Statistical Analysis}

Data were analyzed using SPSS 11.0 software. The $\chi^{2}$ test was used to compare categorical variables between two groups, and the $t$ test, to compare means of continuous variables. Kaplan-Meier survival analysis was used to compare between c-KITpositive and -negative cases.

\section{RESULTS}

Of the 49 patients (38 males and 11 females) included in the study, median age at diagnosis was 45 years (mean: 42 y; range: $10-76$ y). All the pa- 
tients without evidence of disease at the end of treatment had more then 2 years of follow-up. Maximal follow-up was 21 years; median follow-up was 5 years for the entire group and 10 years for the patients who are still alive. Only two patients had recurrent disease and are still alive. One patient is alive without disease 3 years after resection of a single lung metastasis. Anther patient had bone metastases 3 months after completing radiotherapy treatment and is still alive 2 years from the initial diagnosis.

Demographic details and tumor characteristics are seen in Table 1. Most patients $(67 \%(23 / 37)$ of those with known staging) were in Stage IV. The common histology was undifferentiated carcinoma (67\%), and most of the patients had positive EBV status (90\%). Median survival for the entire group is 11.5 years.

\section{HER2}

None of the cases showed positive staining.

\section{C-KIT}

C-KIT expression was absent in normal nasopharyngeal epithelial cells when present in the slides. Infiltrating mast cells scattered in the stroma showed strong c-KIT membrane staining, providing an internal positive control for most cases. Sixteen of the 49 (33\%) specimens were positive for c-KIT.

The majority of the positive tumor cells showed diffuse cytoplasmic staining, some with membranous accentuation (Fig. 1). In addition, three other

TABLE 1. Patient Demographics and Tumor Characteristics According to C-KIT

\begin{tabular}{|c|c|c|}
\hline Characteristic & $\begin{array}{c}\text { Number of } \\
\text { Patients }\end{array}$ & $\begin{array}{l}\text { Number (\%) } \\
\text { C-KIT Positive }\end{array}$ \\
\hline \multicolumn{3}{|l|}{ Gender } \\
\hline Male & 38 & $11(29)$ \\
\hline Female & 11 & $5(45)$ \\
\hline \multicolumn{3}{|l|}{ Ethnic origin } \\
\hline Jewish & 38 & $10(26)$ \\
\hline Arabic & 11 & $6(55)$ \\
\hline \multicolumn{3}{|l|}{ Age at diagnosis } \\
\hline$\leq 21$ & 10 & $7(70)$ \\
\hline $22-59$ & 32 & $9(28)$ \\
\hline$\geq 60$ & 7 & 0 \\
\hline \multicolumn{3}{|l|}{ WHO type } \\
\hline 1 (keratinizing) & 5 & 0 \\
\hline 2 (non-keratinizing) & 11 & $3(27)$ \\
\hline 3 (undifferentiated) & 33 & $13(39)$ \\
\hline \multicolumn{3}{|l|}{ EBV status (EBER) } \\
\hline Positive & 44 & $16(36)$ \\
\hline Negative & 5 & 0 \\
\hline \multicolumn{3}{|l|}{ Stage } \\
\hline 1 & 1 & 0 \\
\hline 2 & 5 & $2(40)$ \\
\hline 3 & 8 & $4(50)$ \\
\hline $4 a$ & 10 & $3(30)$ \\
\hline $4 b$ & 6 & $1(17)$ \\
\hline $4 \mathrm{c}$ & 7 & $2(29)$ \\
\hline Unknown & 12 & $4(33)$ \\
\hline
\end{tabular}

specimens had weak positive staining of $<10 \%$ of the tumor cells and thus were considered as negative. There was no case with positive staining of $10-30 \%$ of tumor cells. Seven specimens showed positive staining of $30-50 \%$ of the tumor cells. Nine specimens had strongly positive staining of $>50 \%$ of the tumor cells (Fig. 1).

\section{Correlation between Clinical Data and Positive C-KIT Staining}

The patients with c-KIT-positive staining were statistically younger than the negative-staining patients (mean age, $29 \pm 14$ y versus $47 \pm 16 \mathrm{y} ; P=$ $.001)$. Survival was correlated to age $(P<.015)$. Although patients with c-KIT expression showed survival advantage over those without (Fig. 2), the difference was not statistically significant. The number (\%) of c-KIT-positive patients in each clinical and pathological category can be seen in Table 1. Only patients with undifferentiated or nonkeratinizing carcinoma showed c-KIT-positive staining, whereas the five samples of squamous carcinoma were negative. Only positive EBV samples had cKIT-positive staining, whereas the five samples of negative EBV status were negative. In other categories, no statistically significant difference was detected between patients with/without c-KIT expression. Median stage of the c-KIT-negative patients was $4 \mathrm{a}$, whereas median stage of the c-KIT-positive patients was 3 to $4 \mathrm{a}$ (NS). There were c-KIT-positive patients in all stages and four such patients in the group of unknown-stage patients.

\section{DISCUSSION}

HER2 (c-erbB-2) is a proto-oncogene that encodes a human epidermal cell growth factor receptor-like protein (6). The pivotal publication in 1987 by Slamon and colleagues (7) demonstrated that HER2 gene amplification independently predicted overall survival and disease-free survival in node-positive breast cancer patients. Since then, HER2 as a prognostic or predictive factor has been the focus of intensive study. Prognostic value has been found in some other cancers, such as ovarian, non-small cell lung, gastric (8-10), and others, although clinical benefit from treatment with trastuzumab has not been found in cancers other than breast carcinoma. The role of HER2 as a prognostic marker in NPC is controversial, and even the finding of oncogene amplification or oncoprotein overexpression is unsettled. In the study by Roychowdhury et al. (11), in which either cytoplasmic or membranous staining were defined as positive HER2 overexpression, $46 \%$ of the cases were positive, with correlation to disease-free survival and overall survival. It is not clear from the study how 


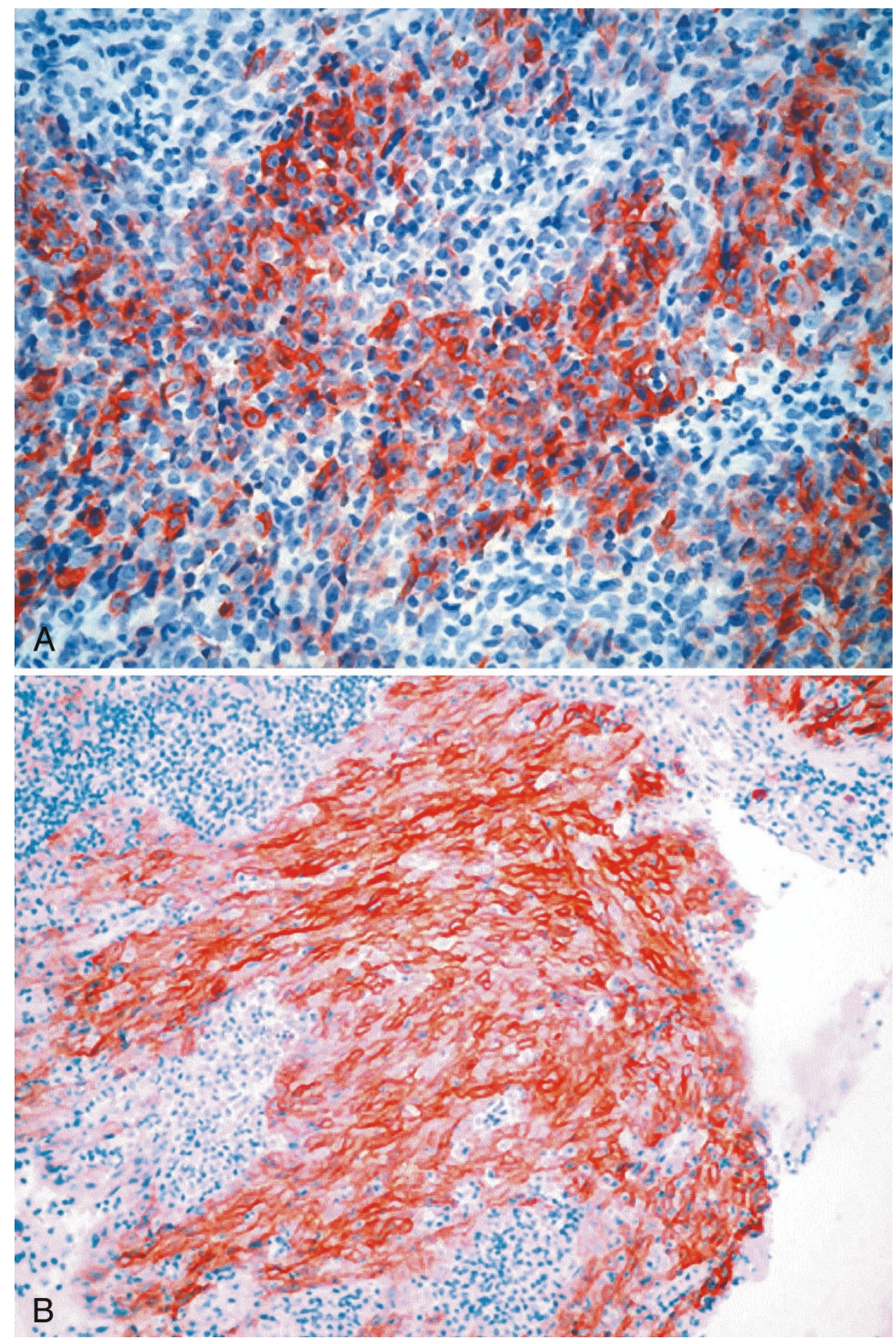

FIGURE 1. A, diffuse c-KIT staining of the tumor cells. The stromal cells, lymphocytes, and plasma cells were not stained. The staining is cytoplasmatic with membranous accentuation in some cells. B, strongly diffuse c-KIT staining of the tumor cells. In this case, the membranous accentuation is prominent.

many positive cases had membranous staining, and the monoclonal antibody that was used is not in regular use for HER2 staining in breast cancer cases. We considered only +3 or +2 positive membranous staining as overexpression, following the criteria used for breast carcinoma. In a study by Yan et al. (12), $20 \%$ of the cases showed HER2 overexpression $(+2$ or +3$)$ by immunohistochemical analysis, but none had gene amplification by fluorescence in situ hybridization (FISH). Similar negative results, using the same immunohistochemical staining as in our study, were found by Shnayder $e t$ 


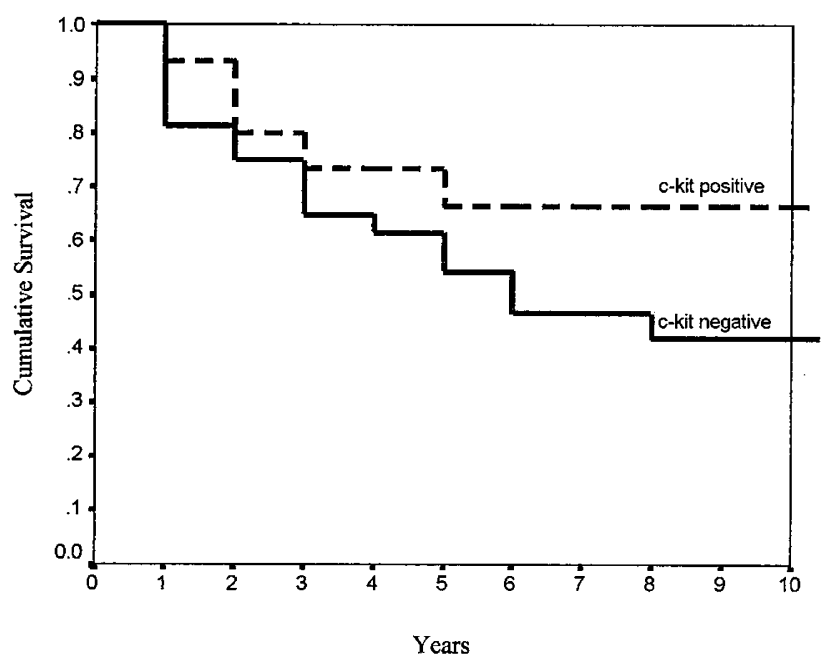

FIGURE 2. Kaplan-Meier estimates of overall survival of c-KIT-positive and -negative cases.

al. (13). The only HER2 gene amplification, using polymerase chain reaction (PCR) with correlation to $\mathrm{T}$ stage, was found by Yazici et al. (14). The meaning of their finding is not clear, as PCR is not used routinely in HER2 gene amplification studies in breast cancer patients.

The c-KIT proto-oncogene codes for a Type III tyrosine kinase receptor protein (CD117) that is structurally related to other transmembrane receptors, including platelet-derived growth factor and colony-stimulating factor $1(15,16)$. Expression of CD117 in normal human tissues has been reported to be strongly positive (mostly membranous) in mast cells and germ cells, and moderately positive (cytoplasmic) in breast ductal epithelium, some renal tubules, gastric parietal cells, melanocytes, and ovarian cells (17). There are no data about the expression of CD117 in normal head and neck tissues. In the large study by Arber et al. (17), 576 human tumor tissues were studied for c-KIT (CD117) expression. Mast cell disease showed diffuse strong positive staining. Of the other examined tumors, some of the seminoma germ cell tumors, serous ovarian carcinoma, and melanoma showed a moderately to strongly positive expression of CD117. Other studies found strong positive staining in some childhood sarcomas (22), angiomyolipoma (23), and adenoid cystic carcinoma (24). In GIST, it was recently reported that c-KIT activation occurs in all cases, regardless of the mutational status of c-KIT (25). To the best of our knowledge, the current study is the first to look for CD117 expression in NPC. To avoid non-specific overstaining of tumor cells, as noted by Hornick and Fletcher (26), we did not use heat-induced antigen retrieval in our study before staining the slides for c-KIT. In previous studies, several cutoff levels of c-KIT expression were used, ranging from 10 (27) to $20 \%$ (28), $25 \%$
(23), and even 50\% (24). Although we used a cutoff level of $10 \%$, all our positive cases had staining of $>30 \%$ of cells, and the staining intensity of the three cases with $<10 \%$ of cells was weak. Further studies are needed to determine the best cutoff level that correlates with clinical parameters.

According to the current study, patients with cKIT-positive tumors were younger than the c-KITnegative cases: 7 of 10 children but none of the $60+$ patients were c-KIT positive. Most of the NPC patients in Israel, as found in our study also, have undifferentiated carcinoma, EBV-associated (2). All patients with squamous histology and patients with negative EBER were c-KIT negative. However, as the total number of cases was small, a larger study including greater numbers of patients with squamous carcinoma histology or EBV-negative status is needed to validate these results. Although a trend for better survival was seen for positive c-KIT patients, it did not reach statistical significance. c-KIT expression did not add prognostic value to known prognostic factors (stage and histology) in the current study, but a larger study is warranted to test its possible value. It could be that the study group was too small to show the possibility of c-KIT being a positive prognostic factor and that a larger group study is needed. Because immunohistochemical analysis cannot identify tumors as KIT-signaling driven (29), demonstration of KIT activation by molecular analysis is recommended for the optimal use of tyrosine kinase inhibitors in clinical practice.

\section{CONCLUSION}

c-KIT protein was expressed in one third of the NPC patients in the current study, only in EBVpositive undifferentiated or nonkeratinizing carcinoma cases. Further research is needed to identify c-KIT DNA mutations or c-KIT activation and to test the possibility of treatment with imatinib mesylate. HER2 protein was not found in the same tumors.

Acknowledgment: The authors thank Mrs. M. Perlmutter for her help in the preparation of this paper.

\section{REFERENCES}

1. Parkin DW, Pisani P, Ferlay J. Estimates of the worldwide incidence of 25 major cancers in 1990. Int J Cancer 1999;80: 827-41.

2. Kuten A, Cohen Y, Lavie R, et al. Update on nasopharyngeal carcinoma in northern Israel. Strahlenther Onkol 1994;170: 565-70.

3. Lee AW, Poon YF, Foo W, et al. Retrospective analysis of 5037 patients with nasopharyngeal cancer treated during 19761985: overall survival and patterns of failure. Int J Radiat Oncol Biol Phys 1992;23:261-70. 
4. Sham JS, Choy D, Choi PH. Nasopharyngeal carcinoma: the significance of neck node involvement in relation to the pattern of distant failure. Br J Radiol 1990;63:108-13.

5. Al-Sarraf M, LeBlanc M, Giri S, et al. Chemoradiotherapy versus radiotherapy in patients with advanced nasopharyngeal cancer: phase III randomized intergroup study 0099. J Clin Oncol 1998;16:1310-7.

6. Dowsett M, Cooke T, Ellis I, et al. Assessment of HER2 status in breast cancer: why, when and how? Eur J Cancer 2000;36: $170-6$.

7. Slamon DJ, Clark GM, Wong SG, et al. Human breast cancer: correlation of relapse and survival with amplification of the Her-2/neu oncogen. Science 1987;235:177-82.

8. Gershenson DM, Tortolero-Luna G, Malpica A, et al. Ovarian intraepithelial neoplasia and ovarian cancer. Obstet Gynecol Clin North Am 1996;23:475-543.

9. Allgayer H, Babic R, Gruetzner KU, et al. c-erbB-2 is of independent prognostic relevance in gastric cancer and is associated with the expression of tumor-associated protease systems. J Clin Oncol 2000;18:2201-9.

10. Smit EF, Groen HJ, Splinter TA, et al. New prognostic factors in resectable non-small cell lung cancer. Thorax 1996;51: 638-46.

11. Roychowdhury DF, Tseng A Jr, Fu KK, et al. New prognostic factors in nasopharyngeal carcinoma. Tumor angiogenesis and c-erbB2 expression. Cancer 1996;77:1419-26.

12. Yan J, Fang Y, Huang BJ, et al. Absence of evidence for HER2 amplification in nasophryngeal carcinoma. Cancer Genet Cytogenet 2002;132:116-9.

13. Shnayder Y, Kuriakose MA, Yee H, et al. Adhesion molecules as prognostic factors in nasopharyngeal carcinoma. Laryngoscope 2001;111:1842-6.

14. Yazici H, Altun M, Alatli C, et al. c-erbB-2 gene amplification in nasopharyngeal carcinoma. Cancer Invest 2000;18: $6-10$.

15. Besmer P, Murphy JE, George PC, et al. A new acute transforming feline retrovirus and relationship of its oncogene v-kit with the protein kinase gene family. Nature 1986;320: 415-21.

16. Qiu F, Ray P, Brown K, et al. Primary structure of c-kit relationship with the CSF-1/PDGF receptor kinase family oncogenic terminus. EMBO J 1988;7:1003-11.

17. Arber DA, Tamayo R, Weiss LM. Paraffin section detection of the c-kit gene product (CD117) in human tissues: value in the diagnosis of mast cell disorders. Hum Pathol 1998;28: 498-504.

18. Miettinen M, Lasota J. Gastrointestinal stromal tumors: definition clinical histological immunohistochemical and molecular genetic features and differential diagnosis. Virchows Arch 2001;438:1-12.

19. Bokemeyer C, Kuczyk MA, Dunn T, et al. Expression of stem-cell factor and its receptor c-kit protein in normal testicular tissues and malignant germ-cell tumours. J Cancer Res Clin Oncol 1996;122:301-6.

20. Vogel CL, Cobleigh MA, Tripathy D, et al. Efficacy and safety of trastuzumab as a single agent in first-line treatment of HER2-overexpressing metastatic breast cancer. J Clin Oncol 2002;20:719-26.

21. Demetri GD, Von-Mehren M, Blanke CD, et al. Efficacy and safety of imatinib mesylate in advanced gastrointestinal stromal tumors. N Engl J Med 2002;347:472-80.

22. Smithey BE, Pappo AS, Hill DA. c-kit expression in pediatric solid tumors. A comparative immunohistochemical study. Am J Surg Pathol 2002;26:486-92.

23. Makhlouf HR, Remotti HE, Ishak KG. Expression of KIT (CD117) in angiomyolipoma. Am J Surg Pathol 2002;26:493-7.

24. Holst VA, Marshall CE, Moskaiuk CA, et al. KIT protein expression and analysis of c-kit gene mutation in adenoid cystic carcinoma. Mod Pathol 1999;12:956-60.

25. Rubin BP, Singer S, Tsao C, et al. KIT activation is a ubiquitous feature of gastrointestinal stromal tumors. Cancer Res 2001;61:8118-21.

26. Hornick JL, Fletcher CDM. Immunohistochemical staining for KIT (CD117) in soft tissue sarcomas is very limited in distribution. Am J Clin Pathol 2002;117:188-93.

27. Sabah M, Leader M, Kay E. The problem with KIT: clinical implications and practical difficulties with CD117 immunostaining. Appl Immunohistochem Mol Morphol 2003;11:5661.

28. Lonardo F, Pass HI, Lucas DR. Immunohistochemistry frequently detects c-Kit expression in pulmonary small cell carcinoma and may help select clinical subsets for a novel form of chemotherapy. Appl Immunohistochem Mol Morphol 2003;11:51-5.

29. Miettinen M. New challenges in the identification of gastrointestinal stromal tumors and other possible KIT-driven tumors. Am J Clin Pathol 2002;117:183-5. 